\title{
Stress-Induced Changes in Closure Domain Structure Dynamics in Bistable Ferromagnetic Microwire
}

\author{
J. Onufer*, J. Ziman, M. Kladivová \\ Department of Physics, Technical University of Košice, Letná 9, 04200 Košice, Slovakia
}

\begin{abstract}
A new experimental method for the study of closure domain structure dynamics in bistable ferromagnetic microwires is proposed. The simple experimental set-up enables detection of the presence of a free domain wall in the wire after application of a well-defined rectangular magnetic field pulse. The changes in closure domain structure dynamics, caused by applied tensile stress, are demonstrated by measurements on $\mathrm{Fe}_{77.5} \mathrm{~B}_{15} \mathrm{Si}_{7.5}$ glasscoated microwire. Minimum critical field for the release of a domain wall from the closure domain structure increases with increasing stress, while on the other hand, the minimum time needed for this process rapidly decreases with increasing stress.
\end{abstract}

DOI: 10.12693 /APhysPolA.126.80

PACS: $75.30 . \mathrm{Gw}, 75.50 . \mathrm{Kj}, 75.60 . \mathrm{d}, 75.60 . \mathrm{Ch}, 75.60 . \mathrm{Ej}, 75.60 . \mathrm{Jk}$

\section{Introduction}

Fe-based glass-coated amorphous ferromagnetic microwires are interesting materials due to their potential for technical applications [1, 2]. Magnetic bistability is one of the characteristic features of these high magnetostrictive microwires. They are ideal materials for the study of single domain wall dynamics [3-6]. The domain structure of amorphous glass-coated microwires with positive magnetostriction consists of one large axial domain surrounded by radial domains [3]. A closure domain structure, which minimizes the stray field, appears at each end of the microwire. Typically magnetization reversal starts by depinning a single domain wall from the closure structure, which then propagates along the microwire. In this paper a new simple experimental method which allows study of the dynamics of the process of wall depinning from the wire end is proposed. The critical parameters of a rectangular magnetic field pulse (its length and magnitude) [6] at which a free domain wall is released from the wire end are determined from this experiment. The influence of applied tensile stress on the closure domain structure dynamics is also studied using this method.

\section{Experiment}

Measurements were performed on an as-cast amorphous ferromagnetic glass-coated $\mathrm{Fe}_{77.5} \mathrm{~B}_{15} \mathrm{Si}_{7.5} \mathrm{mi}-$ crowire with metallic nucleus diameter of about $15 \mu \mathrm{m}$ and glass layer thickness of about $7 \mu \mathrm{m}$. The length of the sample used in the experiment was about $4 \mathrm{~cm}$ (Fig. 1).

The experimental set-up, shown in Fig. 2, consists of a thin magnetizing coil with a length of $3 \mathrm{~cm}$ and radius of $1 \mathrm{~mm}$ and two pick-up coils for detection of domain wall propagation. The magnetizing coil (MC) is connected to a function generator and the parameters of this coil allow creation of a well-defined rectangular magnetic field

*corresponding author; e-mail: jozef .onufer@tuke.sk pulse of magnitude $H_{m}$ and length $\tau$. Pick-up coils are connected to a digital oscilloscope in such a way that voltages of opposite signs are induced due to wall propagation. One end of the wire is outside the MC, to prevent depinning of the wall from this end. Tensile stress can be applied by a mechanical load attached to it. The end of the wire located inside the MC was fixed.

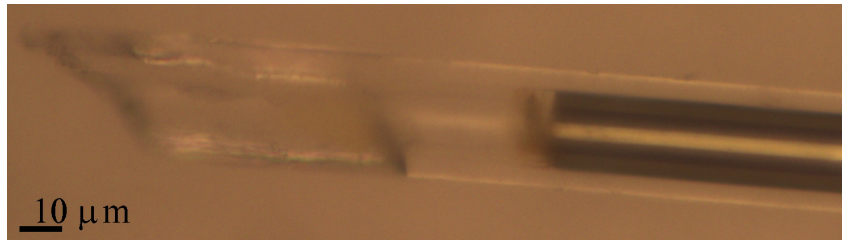

Fig. 1. Optical microscopy image of an etched wire end.

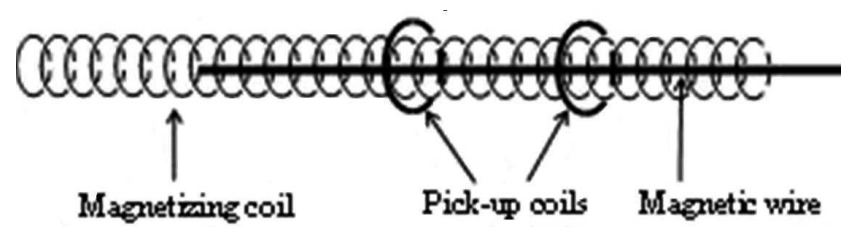

Fig. 2. Experimental set-up.

In one measuring cycle a series of four pulses are generated by the MC. The first pulse is long $(\tau>1 \mathrm{~ms})$ and its magnitude is high enough to magnetize the wire in negative direction. The second pulse has positive sign with magnitude $H$ and length $\tau_{c}$. The third pulse is long ( $\tau>1 \mathrm{~ms}$ ), has positive sign and its magnitude is high enough to move a free wall along the wire, but not high enough to depin the wall from the wire end [5]. The fourth pulse is long ( $\tau>1 \mathrm{~ms})$ and its magnitude is high enough to magnetize the wire in positive direction. If the second pulse causes depinning of a domain wall from the wire end, after the third pulse the whole wire is magnetized in positive direction and no wall propaga- 
tion is registered by the pick-up coils during the fourth pulse. If the second pulse does not cause the generation of a free domain wall, then the wire remains magnetized in negative direction also after the third pulse and wall propagation is registered by the pick-up coils during the fourth pulse. By this procedure it is possible to detect whether the wall was depinned or not during the second pulse. For a given value of $\tau_{c}$ the corresponding critical magnitude of field pulse $H_{p c}$ above which and below which a free domain wall was and was not generated, respectively, was found in a similar way as reported in [6].

\section{Results and discussion}

The experiment described above was carried out on samples with various kinds of wire end treatments: simple cutting, shearing and etching of the wire end in $\mathrm{HCl}$ (see Fig. 1). No significant influence of these treatments on experimental values of critical fields $H_{p c}$ was observed.

The relations between critical pulse parameters for different applied tensile stresses are shown in Fig. 3.

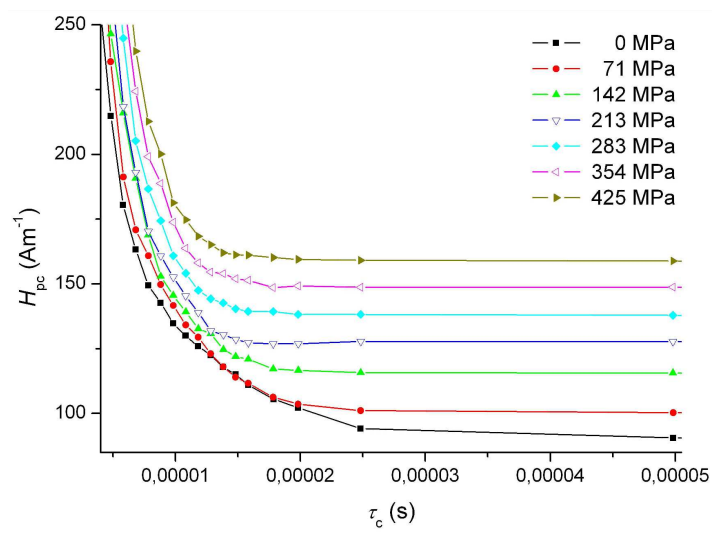

Fig. 3. Experimental dependences $H_{p c}$ vs. $\tau_{c}$ for various values of applied tensile stress.

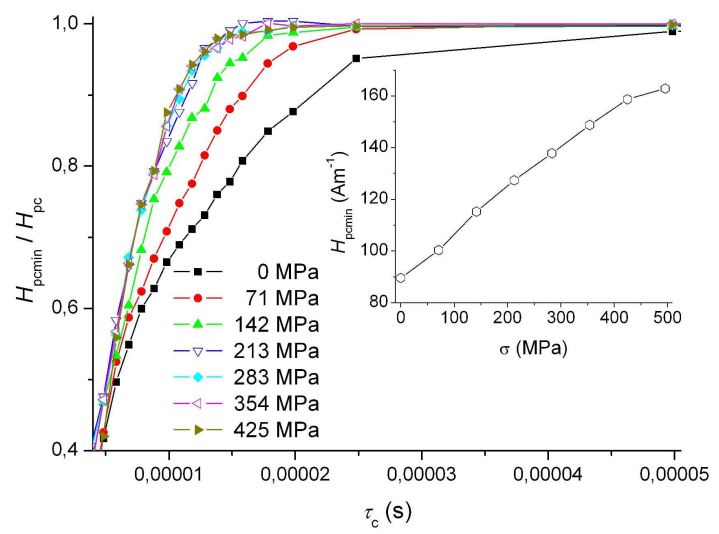

Fig. 4. Inverse normalized magnetic field $H_{p c \text { min }} / H_{p c}$ as a function of pulse length $\tau_{c}$ for various values of tensile stress.

As can be expected the magnitude $H_{p c}$ increases with decreasing $\tau_{c}[6]$. For long time intervals $\tau_{c}$, the critical field $H_{p c}$ converges to its minimum value $H_{p c \text { min }}$. As can be seen in Fig. 3, and also in the inset in Fig. 4, $H_{p c \text { min }}$ increases with the increasing applied stress. This is in agreement with the increase in the switching field with applied stress, typically observed in this kind of microwires [3, 7]. New information which this experiment presents here, concerns the dynamics of the depinning process. The dynamics for different values of applied stress can be analysed using the plot of inverse normalized magnetic field $H_{p c \text { min }} / H_{p c}$ as a function of pulse length $\tau_{c}$ (see Fig. 4). These plots make it possible to determine the length of the field pulse $\tau_{c \text { min }}$ below which $H_{p c \min } / H_{p c}<1$. In other words, it is possible to determine the minimum time interval needed to produce a free domain wall at applied minimum field $H_{p c \text { min }}$. We can see that these time intervals are surprisingly short $\left(\tau_{c \text { min }} \approx 50 \mu \mathrm{s}\right.$ for no applied stress). Moreover, the value of $\tau_{c \text { min }}$ decreases rapidly for low values of applied stresses, and for values higher than about $200 \mathrm{MPa}$ it stops changing. The minimum value of about $15 \mu$ s was obtained. The next step of the processing of experimental data in Fig. 3 can be the modeling of the depinning process and fitting theoretical results to the experimental ones in a similar way as in [6]. This approach is under preparation.

\section{Conclusions}

A new experiment for the study of closure domain structure dynamics in bistable magnetic wires was proposed. The changes in closure domain structure dynamics, caused by applied tensile stress are demonstrated by measurements on $\mathrm{Fe}_{77.5} \mathrm{~B}_{15} \mathrm{Si}_{7.5}$ glass-coated microwire. It was found that treatment of the wire ends has negligible influence on the obtained results. The minimum field for the release of a domain wall from the closure domain structure increases with increasing stress. On the other hand the minimum time needed for this process rapidly decreases with increasing stress. Theoretical modeling of this process can produce more useful information.

\section{Acknowledgments}

This work was supported by the Slovak Research and Development Agency under contract No. APVV-0027-11 and also by VEGA grant No. 1/0778/12.

\section{References}

[1] V. Zhukova, M. Ipatov, A.Zhukov, Sensors 9, 9216 (2009).

[2] D. Praslička, J. Blažek, M. Šmelko, J. Hudák, A.Čverha, I. Mikita, R. Varga, A. Zhukov, IEEE T. Magn. 49, 128 (2013).

[3] M. Vázquez, Handbook of Magnetism and Advanced Mag. Mat. 4 (2007), ISBN: 978-0-470-02217-7.

[4] M. Vázquez, G.A. Basheed, G. Infante, R.P. Del Real, Phys. Rev. Lett. 108, 037201 (2012).

[5] J. Ziman, J. Onufer, M. Kladivová, J. Magn. Magn. Mat. 323, 3098 (2011).

[6] J. Onufer, J. Ziman, M. Kladivová, J. Magn. Magn. Mat. 344, 148 (2013).

[7] E. Komová, M. Varga, R. Varga, P. Vojtaník, Acta Phys. Polonica A 113, 135 (2008). 